\title{
Truncated BID
}

National Cancer Institute

\section{Source}

National Cancer Institute. Truncated BID. NCI Thesaurus. Code C104460.

Truncated BID is encoded by the human BID gene and is a C-terminal peptide that results from caspase- 8 cleavage of the $\mathrm{BH}$-interacting domain death agonist protein. This protein fragment localizes with the mitochondrial membrane and can stimulate the release of cytochrome $\mathrm{c}$ and promote the progression of apoptosis. 